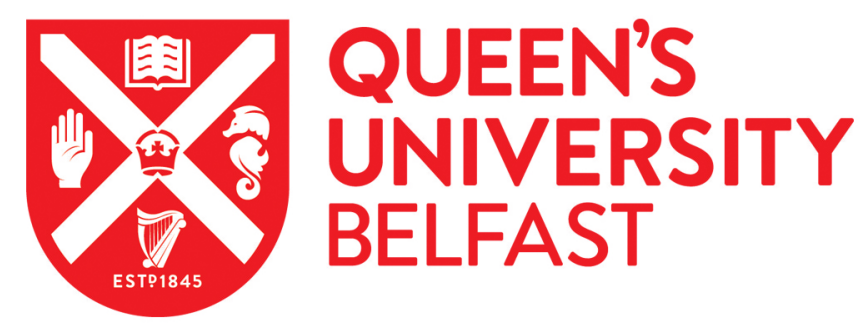

\title{
Pseudomonas aeruginosa in cystic fibrosis patients with c.1652G>A (G551D)-CFTR treated with ivacaftor-Changes in microbiological
} parameters

Millar, B. C., Mccaughan, J., Rendall, J. C., Downey, D. G., \& Moore, J. E. (2018). Pseudomonas aeruginosa in cystic fibrosis patients with c.1652G>A (G551D)-CFTR treated with ivacaftor-Changes in microbiological parameters. Journal of Clinical Pharmacy and Therapeutics, 43(1), 92-100. https://doi.org/10.1111/jcpt.12616

Published in:

Journal of Clinical Pharmacy and Therapeutics

Document Version:

Peer reviewed version

Queen's University Belfast - Research Portal:

Link to publication record in Queen's University Belfast Research Portal

\section{Publisher rights}

(C) 2017 John Wiley \& Sons Ltd. This work is made available online in accordance with the publisher's policies. Please refer to any applicable terms of use of the publisher.

javascript:void(0);

\section{General rights}

Copyright for the publications made accessible via the Queen's University Belfast Research Portal is retained by the author(s) and / or other copyright owners and it is a condition of accessing these publications that users recognise and abide by the legal requirements associated with these rights.

\section{Take down policy}

The Research Portal is Queen's institutional repository that provides access to Queen's research output. Every effort has been made to ensure that content in the Research Portal does not infringe any person's rights, or applicable UK laws. If you discover content in the

Research Portal that you believe breaches copyright or violates any law, please contact openaccess@qub.ac.uk. 


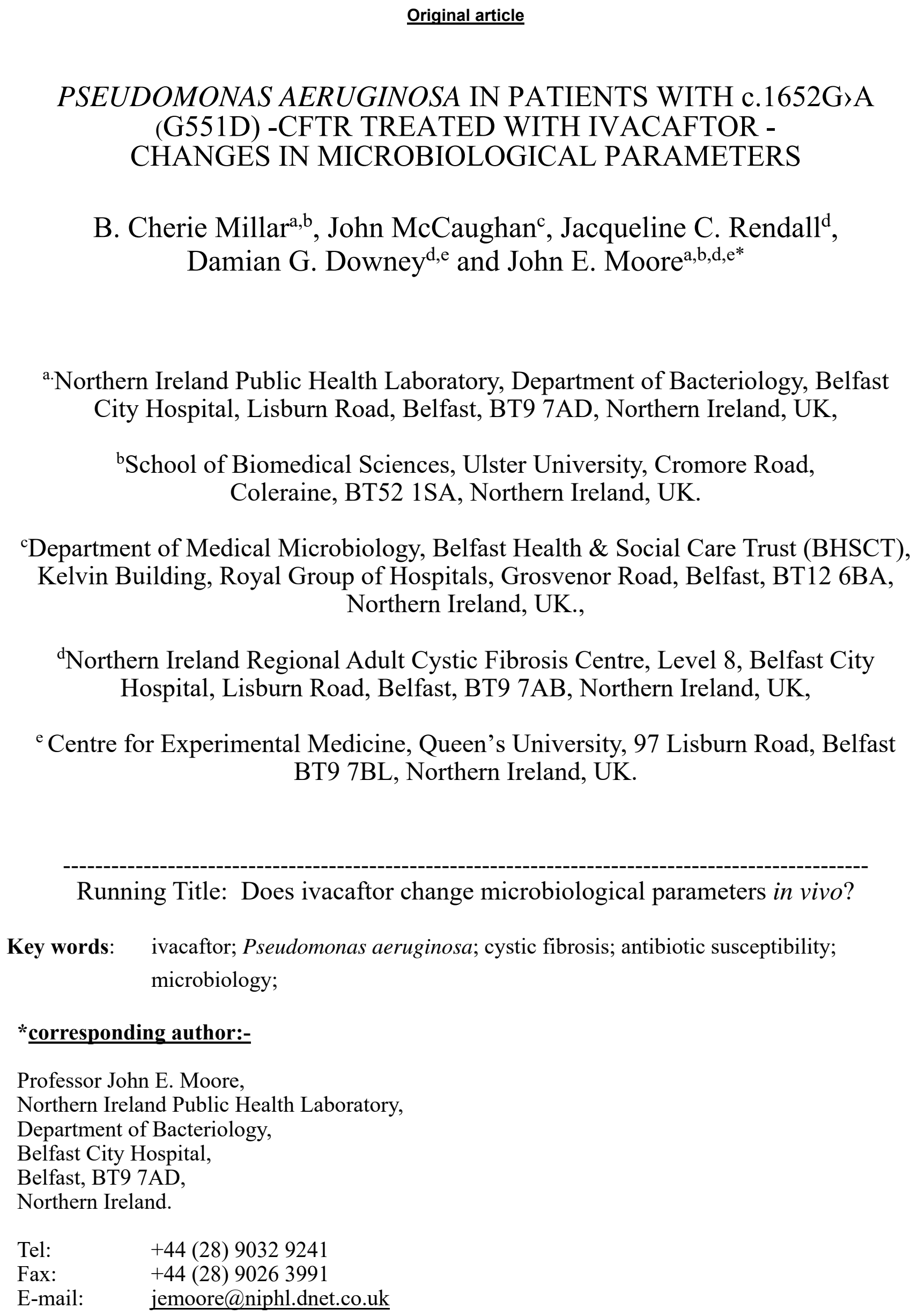

\section{PSEUDOMONAS AERUGINOSA IN PATIENTS WITH c.1652G〉A (G551D) -CFTR TREATED WITH IVACAFTOR - CHANGES IN MICROBIOLOGICAL PARAMETERS}

\author{
B. Cherie Millar ${ }^{\mathrm{a}, \mathrm{b}}$, John McCaughan ${ }^{\mathrm{c}}$, Jacqueline C. Rendall ${ }^{\mathrm{d}}$, \\ Damian G. Downey ${ }^{\mathrm{d}, \mathrm{e}}$ and John E. Moore $\mathrm{e}^{\mathrm{a}, \mathrm{b}, \mathrm{d}, \mathrm{e}^{*}}$
}
a. Northern Ireland Public Health Laboratory, Department of Bacteriology, Belfast City Hospital, Lisburn Road, Belfast, BT9 7AD, Northern Ireland, UK, ${ }^{\mathrm{b} S c h o o l ~ o f ~ B i o m e d i c a l ~ S c i e n c e s, ~ U l s t e r ~ U n i v e r s i t y, ~ C r o m o r e ~ R o a d, ~}$ Coleraine, BT52 1SA, Northern Ireland, UK.

cDepartment of Medical Microbiology, Belfast Health \& Social Care Trust (BHSCT), Kelvin Building, Royal Group of Hospitals, Grosvenor Road, Belfast, BT12 6BA, Northern Ireland, UK.,
dNorthern Ireland Regional Adult Cystic Fibrosis Centre, Level 8, Belfast City Hospital, Lisburn Road, Belfast, BT9 7AB, Northern Ireland, UK, BT9 7BL, Northern Ireland, UK.

${ }^{\text {e }}$ Centre for Experimental Medicine, Queen's University, 97 Lisburn Road, Belfast

Running Title: Does ivacaftor change microbiological parameters in vivo?

Key words: ivacaftor; Pseudomonas aeruginosa; cystic fibrosis; antibiotic susceptibility; microbiology;

\section{*corresponding author:-}

Professor John E. Moore,

Northern Ireland Public Health Laboratory,

Department of Bacteriology,

Belfast City Hospital,

Belfast, BT9 7AD,

Northern Ireland.

Tel: $\quad$ +44 (28) 90329241

Fax: $\quad+44(28) 90263991$

E-mail: $\quad$ jemoore@niphl.dnet.co.uk 
52 What is known and objective: The CFTR potentiator, Ivacaftor (IVA), has been widely used in the

53 treatment of cystic fibrosis (CF) patients with the G551D mutation. To date, there has been limited

54 information on the microbiological status of patients on this therapy and no data on the effect (if any)

55 on the in vivo antibiotic susceptibility of Pseudomonas aeruginosa isolated from patients on therapy.

56 Whilst IVA intervention is not designed per se as anti-infective, the effect (if any) of this molecule to

57 CF patients' microbiological status merits careful monitoring. Therefore, it was the aim of this

58 observational study to examine the effect in patients, both before and after commencement of IVA

59 therapy, on several commonly reported microbiological markers in CF patients, including (i) bacterial

60 density, (ii) frequency (rate) of isolation of bacterial pathogens, particularly Pseudomonas aeruginosa

61 and (iii) antimicrobial susceptibility of these isolates to commonly prescribed oral and iv antibiotics.

62 In addition, we wished to examine the requirements for these antibiotics in CF patients, before and

63 after commencement of IVA therapy.

65 Methods: Archived data from 15 adult patients with the c.1652G>A (G551D) mutation were followed

66 from two years pre-IVA therapy to two years after commencement of IVA therapy. The

67 microbiological parameters examined included (i) oral antibiotic courses taken, (ii) intravenous (iv)

68 antibiotic courses taken, (iii) rate of isolation of non-mucoid Pseudomonas aeruginosa (NM-PA) and

69 mucoid Pseudomonas aeruginosa (M-PA), (iv) density of NM-PA and M-PA and (v) antimicrobial

70 susceptibility of NM-PA and M-PA to 11 antibiotics [aminoglycosides, beta-lactams, polymyxin and

71 fluoroquinolone]

72

73 Results and discussion: Following commencement of IVA therapy, patients required less iv antibiotic

74 courses but no change in number of oral antibiotics courses. There was significant reduction in both

75 the rate of isolation and density of M-PA ( $p=0.02 ; p=0.006$, respectively). In contrast, there was no

76 significant reduction in both the rate of isolation and density of NM-PA $(p=0.90 ; p=0.07$, respectively). 
77 Antimicrobial susceptibility in NM-PA and M-PA was not significantly reduced within any of the

78 antibiotics classes or individual antibiotics examined. Increased susceptibility was noted in the

79 beta-lactam class for NM-PA and M-PA, in particular with ceftazidime.

80

81 What is new and conclusion: Overall, (i) the requirement for less iv antibiotic therapy, (ii) a reduction

82 in the rate and density of M-PA, and (iii) no reduction in antibiotic susceptibility, indicates that

83 microbiological parameters with patients on IVA therapy were not detrimentally affected.

84

85

86

87 
What is known and objective:

89 The dominant feature of cystic fibrosis $(\mathrm{CF})$-related disease is the deterioration in patients' lung

90 function due to the chronic presence of bacterial pathogens, particularly Pseudomonas aeruginosa.

91 Any change in the status of the patient's microbiology may have a significant effect in clinical

92 outcome, in either a positive or negative manner. Outside of pulmonary exacerbation, the relative

93 microbiological stability of the cystic fibrosis (CF) lung is a fine equilibrium of multiple factors. In

94 microbiological terms, these include the presence/absence of bacterial pathogens, numbers of

95 organisms present, antibiotic resistance, carriage/expression of bacterial virulence determinants,

96 relative phase of bacterial growth (e.g. presence of senescent cells/persisters), host/environmental

97 stress responses and relative nutritional/starvation status of bacterial pathogens, iron sequestration and

98 competition and the dynamic flux from the co-habiting microbiome of the lung. Several other host

99 (patient) related factors can also contribute to this instability in this two host [patient \& bacterium]

100 system.

102 In microbiological terms, what is not well understood is the sequencing and interactions of these

103 factors, when they occur together in a particular pattern, what precipitates the tipping of the relatively

104 stable CF lung into a pulmonary exacerbation or alternatively the interactions which drive the CF lung

105 to a more stable situation, thereby reducing infection and exacerbations. In addition, the relative

106 contribution and interaction of the human host undoubtedly plays a significant part in driving this

107 stable equilibrium to a state of relative instability and hence onwards to a potential pulmonary

108 exacerbation.

109

110 The 21 st century is witnessing the development of many new pharmacological interventions in CF, in

111 order to ameliorate the effects of cystic fibrosis transmembrane conductance regulator (CFTR)

112 dysfunction and thus improve patient outcomes. One such intervention is the use of CFTR

113 correctors/potentiators, as an effective intervention, which has revolutionized CF care in those patients

114 with a genetic profile of their alleles which are predicted to benefit from such interventions. ${ }^{1}$ 
115 Ivacaftor [N-(2,4-Di-tert-butyl-5-hydroxyphenyl)-4-oxo-1,4-dihydroquinoline-3-carboxamide]

116 (VX-770; Kalydeco $\left.{ }^{\circledR}\right)$ [IVA] is an orally bioavailable CFTR-potentiator molecule, that is designed to

117 increase the time that activated CFTR channels at the cell surface remain open, in CF patients with

118 specific mutations, including G551D, G1244E, G1349D, G178R, G551S, S1251N, S1255P, S549N, or

119 S549R. IVA therapy with genetically appropriate CF patients has been shown to improve lung

120 function, as well as improve(i) risk of pulmonary exacerbations,(ii) patient-reported respiratory

121 symptoms, (iii) weight, (iv) concentration of sweat chloride [1] and (v) quality of life. ${ }^{2}$

123 Whilst IVA intervention is not designed per se as anti-infective, the effect (if any) of this molecule to

124 CF patients' microbiological status merits careful monitoring. Therefore, it was the aim of this study to 125 examine the effect in patients, both before and after commencement of IVA therapy, on several

126 commonly reported microbiological markers in CF patients, including (i) bacterial density, (ii)

127 frequency (rate) of isolation of bacterial pathogens, particularly Pseudomonas aeruginosa and (iii)

128 antimicrobial susceptibility of these isolates to commonly prescribed oral and iv antibiotics. In

129 addition, we wished to examine the requirements for these antibiotics in CF patients, before and after

130 commencement of IVA therapy. 


\section{Materials and methods}

Patient population

138 A retrospective analysis was conducted on 15 adult patients, who were receiving therapeutic

139 treatment for their cystic fibrosis with oral ivacaftor. Each patient had a confirmed diagnosis of cystic 140 fibrosis, with at least one copy of the G551D mutation. Patients had an age range of 16-43 years and 141 there were nine males (age range: 16 - 43years) and seven females (age range: 18-32 years). Male 142 patients treatment duration ranged from 18-30 months, with a mean treatment duration of 24 months 143 and female patients treatment durations ranged from 18-29 months, with a mean treatment duration of 14423.4 months.

Antibiotic treatment and microbiological parameters analysed

147 Each parameter was examined (i) two years prior to commencement of ivacaftor therapy and (ii). two 148 years post ivacaftor therapy.

150 Archived microbiological data was analysed from the patients' clinical microbiology record file, with 151 particular reference to (i). total Pseudomonas aeruginosa (PA), (ii). non-mucoid (NM) PA and (iii). 152 Mucoid (M) PA. The specific microbiological parameters investigated included (i). incidence of (PA 153 or NM PA or M PA)/respiratory specimen, (ii). relative culture density of PA [NM PA or M 154 PA ]/respiratory specimen, (iii). antibiotic susceptibility.

The rate of isolation of PA was defined as frequency of PA isolated from sputum and was presented as rate/respiratory specimen. The density of PA isolated from respiratory specimen was quantitatively

158 recorded by taking the laboratory semi-quantitative result (i.e. none,,,++++++ ) and converting these 159 values into a fully quantitative value, ranging from $0,1,2$ and 3, respectively, and expressed per 160 respiratory specimen. 
162 Antibiotic susceptibility was defined by employment of the Relative Resistance Index (RRI) ${ }^{3}$ of PA

163 isolates, within the following classes of antibiotics (agents analysed): aminoglycosides (gentamicin, 164 tobramycin, amikacin); $\beta$-lactams (temocillin, ceftazidime, piperacillin/tazobactam, aztreonam), 165 carbapenems (imipenem, meropenem), polymyxin (colistin) and fluoroquinolone (ciprofloxacin). 166 Relative Resistance Index (RRI) values were calculated for each NM-PA and M-PA isolate in each 167 patient, at each visit to the CF Unit, as either an in-patient or out-patient. Antibiotic susceptibility was 168 routinely recorded as sensitive (S), intermediate (M) or resistant (R). Subsequently, for the purposes 169 of this analysis, RRI values were assigned, as follows: a value of 1 for sensitivity, 2 is 170 intermediate/moderate resistance and 3 for resistant.

172 Additionally, the number of antibiotic courses, both oral and intravenous (IV), were analysed and 173 expressed as the mean courses of antibiotics given per month over the two years prior to 174 commencement of ivacaftor therapy and the two years after commencement of ivacaftor therapy.

176 Statistical analysis

177 Student t-tests were used to compare pre-ivacaftor and post-ivacaftor microbiology findings. Where 178 unpaired t-tests were employed, $\mathrm{F}$ values were calculated to determine equal or unequal variance. $\mathrm{P}$ 179 values and confidence intervals (CIs) were two-sided, where $\mathrm{p} \leq 0.05(5 \%)$ was deemed significant. 180 Error bars were calculated as \pm standard error of the mean (SEM). 


\section{Results}

Antibiotic therapy

There was no significant difference between the number of oral antibiotic courses/month before and after IVA therapy $(0.1 \vee 0.07 ; \mathrm{p}=0.41)$ (Figure 1$)$. There was a significant reduction in the number of iv antibiotic courses/month, following IVA therapy $(0.15 \mathrm{v} 0.02 ; \mathrm{p}=0.0003)$ (Figure 2$)$. There was no requirement for iv antibiotics in 6/15 (40\%) patients, both prior to and following commencement of IVA therapy. Five of the patients who required iv antibiotics pre-IVA therapy (33.3\%) subsequently did not require iv antibiotics, following commencement of IVA therapy. For the remainder of the patients, 4/15 (26.7\%) required less courses of iv antibiotics following commencement of IVA therapy.

All patients who were taking nebulized anti-pseudomonal antibiotics for suppressive therapy continued to take these after commencement of IVA therapy and thus there was no change in the rate of inhaled antibiotics with the Pseudomonas-positive patients.

Rate \& density of Pseudomonas aeruginosa isolation from respiratory specimens

\section{Non-mucoid Pseudomonas aeruginosa}

Eleven patients from the total 15 patients included in this study were culture-positive for NM-PA, prior to commencement of IVA therapy. Overall at a population level, there was no significant 204 difference in either the rate $(p=0.90)$ or density ( $p=0.07)$ of NM-PA, following commencement of IVA therapy (Table 1). When examined statistically, at individual patient level, the density of NM-PA was significantly lower (before $\mathrm{v}$ after commencement IVA therapy) in one patient $(\mathrm{p}=0.02)$, even though this patient had a constant rate of NM-PA isolation (Figure 3). One patient acquired an NM-PA after commencement of IVA therapy, but this isolate appeared to be transient, as it was only reported in a 
three patients remained NM-PA free.

Mucoid Pseudomonas aeruginosa

213 Nine patients in this study were culture-positive for M-PA, prior to commencement of IVA therapy. 214 Overall, at a population level, there was a significant lowering in both the rate $(\mathrm{p}=0.02)$ and density $215(\mathrm{p}=0.006)$ of M-PA, following commencement of IVA therapy (Table 1 \& Figure 4). Interestingly, a 216 reduced density of M-PA isolation was statistically observed in three of the nine patients (33.3\%) and 217 additionally two patients became negative for M-PA after commencement of IVA therapy. None of 218 the six patients previously negative for M-PA prior to IVA therapy gained M-PA, after 219 commencement of therapy.

\section{Other microorganisms}

222 There was no significant difference in both the rate $(\mathrm{p}=0.37)$ and density $(\mathrm{p}=0.43)$ of Staphylococcus aureus in patients $(\mathrm{n}=7)$, before $\mathrm{v}$ after commencement of IVA therapy. Additionally, in one patient, there was no significant difference in the rate or density of Burkholderia cenocepacia, before $\mathrm{v}$ after commencement of IVA therapy. For other non-Pseudomonas organisms, Table 2 details a qualitative comparison of microorganisms cultured in patients before and after commencement of IVA therapy.

\section{Antibiotic susceptibility in PA isolates}

The Relative Resistance Index $[\mathrm{RRI}]^{3}$ was employed as a semi-quantitative method to determine the susceptibility of NM-PA and M-PA. In total, 366 isolates of PA were analysed for antibiotic susceptibility against 11 antibiotics, including 220 NM-PA $(n=109 \& n=111$ before and after commencement of IVA therapy, respectively), as well as $146 \mathrm{M}-\mathrm{PA}(\mathrm{n}=77 \& \mathrm{n}=69$ before and after commencement of IVA therapy, respectively). This equated to approximately 9-10 PA isolates per patient before, as well as after commencement of IVA therapy. 
23711 antibiotics within four antibiotic classes. Figure $5 \mathrm{~b}$ shows antibiotic susceptibility, as expressed as 238 RRI, for NM-PA and Figure 5c for M-PA.

240 There were no statistically significant differences in antibiotic susceptibility for the aminoglycosides, 241 polymyxin (colistin) or fluoroquinolone (ciprofloxacin) in NM-PA and M-PA (Figure 5a-c). Whilst 242 Figure 5a-c shows a general lowering of the RRI value, this was only statistically significant in one 243 class of antibiotics, namely certain $\beta$-lactam antibiotics, before and after commencement of IVA 244 therapy. For total PA, there is a significant increase in susceptibility relating to ceftazidime $245(\mathrm{p}=0.002)$, tazocin $(\mathrm{p}=0.004)$, azteonam $(\mathrm{p}=0.045)$ and meropenem $(\mathrm{p}=0.005)$. Regarding NM-PA, 246 three $\beta$-lactams showed a significant increase in susceptibility, namely ceftazidime $(\mathrm{p}=0.03)$, tazocin $247(p=0.002)$ and meropenem $(p=0.02)$, whilst only ceftazidime showed a significant increase in 248 susceptibility $(\mathrm{p}=0.04)$, with M-PA (see Figure 5a-c). 


\section{Discussion}

254 With any novel non-antimicrobial pharmacological intervention in CF, it is important to carefully 255 monitor the microbiological status of patients on therapy, in order to ensure that the intervention is not 256 having a deleterious effect on the patients' microbiological parameters, which could potentially manifest in clinical deterioration. In this observational study, our objective was to retrospectively examine the microbiology of CF patients, who had received IVA therapy. This was achieved by examining microbiological data routinely gathered as part of the patient CF care pathway, from two years proceeding initiation of IVA therapy and continuing to approximately two years after commencement of IVA.

Overall, there was a significant reduction in the requirement for iv antibiotic courses when patients commenced IVA therapy $(\mathrm{p}=0.0003)$ (Figure 2). Of the nine patients who required iv antibiotics, prior to the commencement of IVA therapy, five of these did not require any iv antibiotics, whilst on IVA therapy and the remaining four patients required a reduced number of iv antibiotic courses. We did not observe any patient, who did not routinely receive iv antibiotics subsequently requiring iv antibiotics whilst on IVA therapy.

In contrast, there was no statistical difference in requirement for oral antibiotics before versus after commencement of IVA therapy $(\mathrm{p}=0.41)$. Nine patients from the 15 patients examined in this study required oral antibiotics, prior to commencement of IVA therapy and seven of these patients continued to require oral antibiotics whilst on IVA therapy. Following commencement of therapy, there were two patients who did not require any oral antibiotics and one patient who did require oral antibiotics on therapy, who did not require oral antibiotics before commencement of IVA.

Similarly, in a recent study from the Czech Republic, a 21\% reduction on antibiotic therapy (per patient-year) was reported $(\mathrm{p}<0.001)$, however it is not clear whether this therapy included oral, ivs or 
279 both. $^{4}$ In our study we observed an overall significant reduction $(86.7 \%)$ in the number of iv

280 antibiotic courses per month and a $20 \%$ reduction in the number of oral antibiotic courses per month, 281 although not statistically significant.

283 The question does however remain from our study, as to why iv antibiotic usage went down, whilst oral usage remained unaltered. One possible explanation is a lower clinical threshold for starting an oral antibiotic in healthier patients versus that for commencement of an iv antibiotic in sicker patients.

Consequently, oral antibiotics may be an interesting first choice for examining antimicrobial stewardship within cystic fibrosis.

There was no significance difference in either the rate of isolation or density of NM-PA after commencement of IVA therapy. This was in contrast to M-PA, where there was a significant reduction 291 in both the rate of isolation and density. To date, there have been no other reports which have divided total PA into NM-PA and M-PA components, however the study by Heltshe et al. reported significant reduction in the percentage of patients with total PA $(p=0.004)$ and M-PA $(p=0.05)$, following 1 year post IVA therapy. ${ }^{5}$ More recently, a further study reported that IVA caused a marked reduction in PA density, which commenced 48 hours post commencement of IVA therapy and which continued in the first year of therapy. Following this, PA density rebounded in 6/7 patients, commencing at day 210 on IVA therapy. ${ }^{6}$

There is a relative paucity of data describing the fate of bacterial numbers in patients commencing IVA therapy. Recently published data from the 2013 NACFC Meeting demonstrated that appropriate patients on ivacaftor had improved mucociliary clearance ${ }^{7}$ and demonstrated that mucociliary clearance increased from $8.5 \pm 1.7 \%$ at baseline to $18.7 \pm 2.3 \%$ and $17.7 \pm 1.7 \%$ at one and three months post-treatment, respectively ( $\mathrm{p}<0.001$ for each comparison to baseline). Dramatic improvements in peripheral lung clearance were also demonstrated $(1.5 \pm 1.8 \%$ vs. $12.2 \pm 2.0 \%, 9.1 \pm$ $2.4 \%$ at baseline, one month and three months, respectively; $\mathrm{p}<0.05$ for each comparison to baseline. 
Current research, which is part of the GOAL observational study, from Sagel's group in Colorado,

307 also presented at the recent NACFC meeting. ${ }^{8}$ Induced sputum was collected pre- and post-ivacaftor 308 treatment in 14 subjects (age $27 \pm 14$ yrs; FEV1 $84 \pm 23 \%$ predicted; 6 females). Sputum bacterial 309 diversity did not change significantly with treatment [Shannon Diversity: mean change (SE) 0.13 $310(0.14), \mathrm{p}=0.34]$. The combined relative abundance (RA) of traditional CF bacterial pathogens 311 including Pseudomonas, Staphylococcus, Stenotrophomonas, Achromobacter, and Burkholderia 312 trended down with treatment [mean change (SE) -13.9 (8.2), $\mathrm{p}=0.11$ ]. Prevotella RA significantly 313 increased with treatment [mean change (SE) 8.8 (3.0), $\mathrm{p}=0.01$ ]. By qPCR, neither total bacterial load 314 changed significantly between paired samples [mean change (SE) $-0.18(0.16) \log 10$ gene 315 copies/mL, $\mathrm{p}=0.28$ ], nor did Pseudomonas load [mean change (SE) -0.76 (0.66) log 10 gene 316 copies $/ \mathrm{mL}, \mathrm{p}=0.27]$. There were no significant changes in any sputum markers of inflammation, 317 including neutrophil elastase activity [mean change (SE) $-0.1(0.1) \log 10 \mu \mathrm{g} / \mathrm{mL}, \mathrm{p}=0.29$ ].

319 On first examination of the Sagel's data, ${ }^{8}$ it would appear that nothing significant happened to the total bacterial counts nor the PA counts, which is counter- intuitive to data of Donaldson, ${ }^{7}$ with improved mucocillary clearance in patients on ivacaftor. The problem here is that the Sagel group measured bacterial numbers with an inappropriate methodology, namely enumeration solely via a molecular means qPCR. Where DNA is the target for qPCR determination, it will count total bacteria in the sputum specimen and consequently this will include both living, as well as dead bacterial cells. Given the persistence of DNA from living or dead bacterial cells, therefore any changes (+ or -) in culturable bacterial numbers would be effectively missed by using this methodology. The consequences of this would thus create an uncertainty as to whether or not the culturable bacteria in 328 CF sputum in patients on ivacaftor remained constant or not.

On considering the dynamics of growth of bacteria and bacterial CF respiratory pathogens in the $\mathrm{CF}$ airways in patients on ivacaftor, the published data regarding bacterial numbers counted either via molecularly $y^{6,8}$ or conventionally-acquired ${ }^{6}$ does not give any indication if such numbers are present 
333 due to (i) bacterial cells being in the stationery phase in a relative state of senescence/dormancy but

334 which remain culturable, (ii). actively metabolising where numbers dying off equals numbers being 335 generated de novo and (iii). have switched phenotypically from culturable sessile \& planktonic 336 vegetative cells to non-culturable persister cells. Scenarios (i) and (ii) would show no significant 337 change in numbers, even though the physiology leading to this point is completely different. In this 338 regard, due to the now functional ciliated escalator, bacteria need to work extremely hard to maintain 339 their numbers constant, i.e. the Red Queen Hypothesis, as per the data of Sagel. ${ }^{8}$

341 The consequences of which modality is happening is profound. In the case of (i) above, bacterial 342 cells are not actively dividing nor dying, therefore they will not have a high metabolic turnover and 343 should not be as visible to the host immune system. However, in the case of (ii) above, whilst there 344 does not appear to be much happening, in terms of changes in bacterial counts, there is an important 345 occult metabolism taking place, which would be very visible to the host's immune system, which 346 would drive inflammatory processes. Therefore, it is import to understand the microbiological mechanisms underpinning the fate of constant bacterial populations within the CF airways in patients on IVA.

To date, there have been no reports on the effect of IVA therapy on antibiotic susceptibility in PA. In our study, overall, there was no decrease in antibiotic susceptibility observed in PA (NM-PA and M-PA) in this study. Four classes of commonly employed antibiotics were examined, namely aminoglycosides, $\beta$-lactams, a polymyxin and a fluoroquinolone. There was no difference in susceptibility with the aminoglycoside, the polymyxin or the fluoroquinolone. It was interesting to note the increase in susceptibility with the $\beta$-lactam class of antibiotics, in particular ceftazidime, tazocin, aztreonam and meropenem. A rationale for observing a signal in only the $\beta$-lactam antibiotics remains uncertain and this is the subject of a further study, which is underway. 
360 are largely of academic interest only. These data did not demonstrate marked shifts in susceptibility

361 which altered the susceptibility designation, from sensitive, intermediate or resistant. Given this, IVA 362 therapy should not construed in any manner in the antibiotic management of CF patients.

363 Importantly, antibiotic susceptibility from patients on IVA therapy is not reduced and therefore IVA 364 therapy does not appear to have a detrimental association with antimicrobial therapy.

366 What is new and conclusions

367 Overall, although the findings of this study cannot directly correlate with the effect of IVA therapy 368 with microbiological parameters, it is of interest to note that microbiological parameters with patients 369 on IVA therapy were not detrimentally affected. Indeed, overall, (i) the requirement for less iv 370 antibiotic therapy, (ii) a reduction in the rate and density of M-PA, and (iii) no reduction in antibiotic 371 susceptibility, suggests an improvement in the microbiology status of patients on IVA therapy. 
376 Acknowledgements:

377

378 This study was supported by Vertex Pharmaceuticals Inc., Boston, USA, through an

379 Investigator-Initiated Study Grant.

380

381 


\section{REFERENCES}

3841 Ramsey BW, Davies J, McElvaney NG, Tullis E, Bell SC, Dřevínek P. et al. A CFTR potentiator 385 in patients with cystic fibrosis and the G551D mutation. N Engl J Med 2011;365:1663-1672. doi: 386 10.1056/NEJMoa1105185.

2 O'Reilly R, Elphick HE. Development, clinical utility, and place of ivacaftor in the treatment of cystic fibrosis. Drug Des Devel Ther 2013;7:929-937. doi: 10.2147/DDDT.S30345.

Resistance Index (RRI) - a Scoring System for Antibiotic Resistance in Pseudomonas aeruginosa. Brit J Biomed Sci 2017 (in press)

4 Fila L, Valentova Bartakova L, Grandcourtova A, Marel M, Drnek R, Bilkova A, et al. Ivacaftor in cystic fibrosis adults: Czech experience with six years of follow-up. Biomed Pap Med Fac Univ Palacky Olomouc Czech Repub 2016;160:276-279. doi: 10.5507/bp.2016.029. aeruginosa in cystic fibrosis patients with G551D-CFTR treated with ivacaftor. Clin Infect Dis 2015;60:703-712. doi: 10.1093/cid/ciu944.

6 Hisert KB, Heltshe SL, Pope C, Jorth P, Wu X, Edwards RM, et al. Restoring CFTR Function 404 Reduces Airway Bacteria and Inflammation in People With Cystic Fibrosis and Chronic Lung 405 Infections. Am J Respir Crit Care Med 2017; 195:1617-1628. doi: 10.1164/rccm.201609-1954OC.

4077 Donaldson SH, Zeman K, Laube B, Corcoran T, Locke LW, Pilewski J, et al. Effect of ivacaftor 408 on mucociliary clearance and mucus rheology in patients with a G551D CFTR mutation. Pediatr 
409 Pulmonol 2013 48, Issue S36:279-280.

410

4118 Sagel SD, Harris JK, Wagner BD, Zemanick ET, Emmett P, Taylor-Cousar JL, et al. Effects of

412 ivacaftor on airway microbiome and inflammation in G551D patients. Pediatr Pulmonol 2013 48, 413 Issue S36:285.

414

415

416

417 
418 Figure \& Table Titles

420 Figure 1: Number of oral antibiotic course per month for patients before and after commencement of IVA therapy

Figure 2: Number of intravenous antibiotic course per month for patients before and after commencement of IVA therapy

Figure 3a: Rate of non-mucoid PA isolation before and after commencement of ivacaftor (IVA) therapy in patients who were chronically colonised prior to the commencement of IVA therapy (IVA) therapy in patients who were chronically colonised prior to the commencement of IVA therapy

Figure 4a: Rate of mucoid PA isolation before and after commencement of ivacaftor (IVA) therapy in patients who were chronically colonised prior to the commencement of IVA therapy

Figure 4b:Density of mucoid PA isolation before and after commencement of ivacaftor (IVA) therapy in patients who were chronically colonised prior to the commencement of IVA therapy

Figure 5: Antibiotic susceptibility, as expressed by Relative Resistance Index, against four classes of antibiotic in (a) Total Pseudomonas aeruginosa, (b) Non-mucoid $P$. aeruginosa and (c) Mucoid $P$. aeruginosa, isolated from patients before and after commencement of ivacaftor therapy.

6 (Abbreviations: IVA= ivacaftor; $\mathrm{M}=$ mucoid; $\mathrm{NM}=$ non-mucoid; $\mathrm{PA}=$ Pseudomonas aeruginosa) 
447 Table 1: Comparison of the rate of isolation and density of non-mucoid and mucoid

Pseudomonas aeruginosa before and after commencement of ivacaftor therapy.

449

450 Table 2: Non-Pseudomonas organisms cultured from CF patients' respiratory specimens before and after commencement of ivacaftor therapy 